\title{
Utilizing Neural Networks for Stocks Prices Prediction in Stocks Markets
}

\author{
Ahmed S. Mahedy \\ Electrical Engineering - computer and control Faculty of Engineering, Suez Canal University, Ismalia, Egypt \\ E-mail: Ahm_mahedy@yahoo.com \\ Abdelazeem A. Abdelsalam and Reham H. Mohamed \\ Electrical Engineering - computer and control Faculty of Engineering, Suez Canal University, Ismalia, Egypt \\ E-mail: \{eng.abdelazeem@gmail.com, riry4mody@yahoo.com\} \\ Ibrahim F. El-Nahry \\ Electrical Engineering Dept., Faculty of Engineering, Port Said University, Port said, Egypt \\ E-mail: fbsu.elnahry@gmail.com
}

Received: 02 November 2019; Accepted: 08 March 2020; Published: 08 June 2020

\begin{abstract}
The neural networks, AI applications, are effective prediction methods. Therefore, in the current research a prediction system was proposed using these neural networks. It studied the technical share indices, viewing price not only as a function of time, but also as a function depending on several indices among which were the opening and closing, top and bottom trading session prices or trading volume. The above technical indices of a number of Egyptian stock market shares during the period from 2007 to 2017, which can be used for training the proposed system, were collected and used as follows: The data were divided into two sets. The first one contained $67 \%$ of the total data and was used for training neural networks and the second contained 33\% and was used for testing the proposed system. The training set was segmented into subsets used for training a number of neural networks. The output of such networks was used for training another network hierarchically. The system was, then, tested using the rest of the data.
\end{abstract}

Index Terms-Backpropagation Neural Network, Egyptian stock market, Stock Prediction.

\section{INTRODUCTION}

Prediction of stock market data is a crucial issue for stock traders. Stock market data have a highly dynamic property due to a conflicting extent of influential factors. The issue has been approached for business interests by observing market forces, making assumptions and recognizing historical data. Increased attention, from academia in the field of predictions, has driven to development of technical and statistical models. In contrast to conventional methodologies practiced by traders, technical methods are confined and restricted to observations of historical data. One of the most recently examined technical methods is Artificial Neural
Networks (ANN). Originating from neural networks, ANNs are mathematical structures originally designed to mimic architecture, fault-tolerance, and learning capability of neural networks existing in the human brain. ANNs have been successfully applied for prediction in the fields related to medicine, engineering and physics. The methods have been suggested for modeling financial time series due to the capability of mapping nonlinear connections as proposed for stock market data. The performance of ANNs has been compared to statistical approaches, representing significantly improved results for increasing complexity in time series. Further factors affecting the performance of ANNs are the choice of architecture, input data, and quantity of data.

In the current model, technical information of stock such was used as Open-close-High-low prices and volume of stock as training input vector of Artificial Neural Network and make the predict price function in many variable not one variable the time[16,17,25]. Also, it divided the training vector to many sets; each set was used to train an independent neural and all output of each neural formed a training set the head neural network which give the output of over All system. The first level of neural networks can train in parallel to reduce overall time of training of neural network. For the concept of the current research, it was tried to predict the price of the stock in the short term future with less Mean Square Error and fastest training time of Neural Network. The main objective was to maximize the profit by trying to increase the capital.

Through the years, the economists investigated many different methods to try and find an optimal way to predict the movement of a stock. Some of them are Japanese candlesticks [1, 15], Monte Carlo Models [2], Binomial Models [3]. However instead of using those traditional methods, the problem of predicting stock prices using machine learning techniques [9] and specifically Artificial Neural Networks was approached 
[4]. Then, it was tried to come up with an optimal trading strategy to maximize the potential profit. The main idea was to model a stock trading day using historical information of the stock, the stock price after one day was tried to be predicted. Also, it was tried to train to and test the current model on historical stock data collected during the period of 2007 to 2017 from The Egyptian stock market.

The paper in hand is organized as follows: literature review in the second section. The third section introduces the proposed back propagation research method for prediction of stock market. The fourth section deals with an illustration for simulation results of all developed controllers. And the last section includes the concluding remarks.

\section{LITERATURE REVIEW}

Trifonov conducted a study on the architecture of a neural network which used four different technical indicators [6]. For UTOMO, improving optimization in stock price predictions can be done using a gradientbased back propagation neural network approach. Using this gradient descent in BPNN method [5]. Lin, proposed a kind of neural network which is called TreNet, a novel end-to end hybrid neural network. [7].

Hiransha presented a paper, in which there were four types of deep learning architectures, Multilayer Perceptron (MLP), Recurrent Neural Networks (RNN), Long Short-Term Memory (LSTM) and Convolutional Neural Network (CNN) [8]. Chen1 (2018), proposed a deep learning method based on Convolutional Neural Network to predict the stock price movement of Chinese stock market [18].

\section{RESEARCH METHOD}

\section{A. Backpropagation Neural Network}

The demonstration of the limitations of single-layer neural networks was a significant factor in the decline of interest in neural networks in the 1970s. The independent discovery by several researchers and widespread dissemination of an effective general method of training a multilayer neural network had a major role in the reemergence of neural networks as a tool for solving a wide variety of problems [14, 24].This training method, known as backpropagation of errors or the generalized delta rule shall be discussed. It is simply a gradient descent method to minimize the total squared error of the output computed by the net [10]. A multilayer neural network with one layer of hidden units (the $\mathrm{Z}$ units) is shown in Figure 1.

The output units (the Y units) and the hidden units also may have biases (as shown in Figure 1. The bias on a typical output unit $\mathrm{Y}_{\mathrm{k}}$ is denoted by $\mathrm{W}_{0 \mathrm{k}}$. The bias on a typical hidden unit $Z_{y}$ is denoted $V_{0 j}$. These bias terms act like weights on connections from units whose output is always 1. These units are shown in Figure 1, but are usually not displayed explicitly. Only the direction of information flow for the feedforward phase of operation is shown. During the back propagation phase of learning, signals are sent in the reverse direction [11].

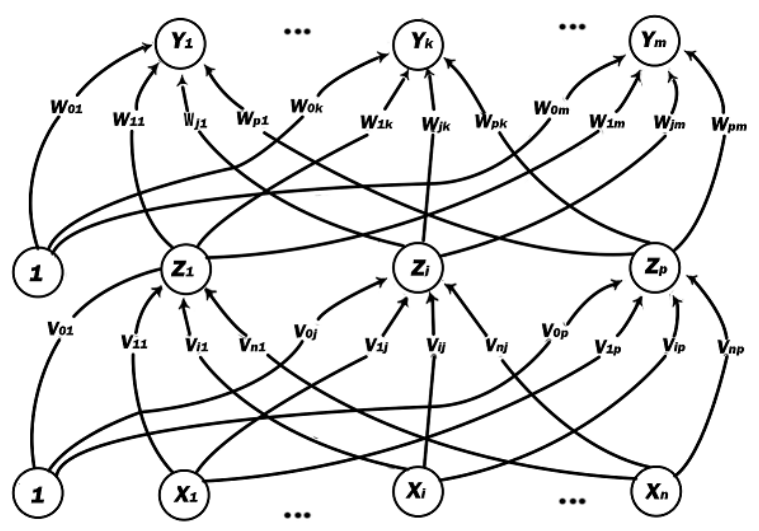

Fig.1. Multilayer neural network with one layer of hidden units

Training a network by backpropagation involves three stages: the feedforward of the input training pattern, the backpropagation of the associated error, and the adjustment of the weights [13].

During feedforward, each input unit $\mathrm{Xi}$ receives an input signal and broadcasts this signal to the each of the hidden units $Z_{1}, \ldots, Z_{p}$. Then each hidden unit computes its activation and sends its signal $\mathrm{z}$ to each output unit. Each output unit $Y_{d}$ computes its activation $y_{k}$ to form the response of the net for the given input pattern. During training, each output unit compares its computed activation $Y_{K}$ with its target value $t_{k}$ to determine the associated error for the pattern with the unit [11].Based on this error, the factor $\delta_{\mathrm{k}}(\mathrm{k}=1 \ldots \mathrm{m})$ is computed $\delta_{\mathrm{k}}$ is used to distribute the error at output unit $\mathrm{Y}_{\mathrm{k}}$ back to all units in the previous layer: the hidden units that are connected to $\mathrm{Y}_{\mathrm{d}}$. It is also used, later, to update the weights between the output and the hidden layer. In a similar manner, the factor $\delta_{\mathrm{j}}(\mathrm{j}=1 \ldots \mathrm{p})$ is ccomputed for each hidden unit $Z_{j}$. It is not necessary to propagate the error back to the input layer, but $\delta_{\mathrm{j}}$ is used to update the weights between the hidden layer and the input layer [11]

After all of the $\delta$ factors have been determined, the weights for all layers are adjusted simultaneously. The adjustment to the weight $\mathrm{W}_{\mathrm{jk}}$ (from hidden unit $\mathrm{Z}_{\mathrm{j}}$ to output unit $Y_{k}$ ) is based on the factor $\delta_{k}$ and the activation $\mathrm{Z}_{\mathrm{j}}$ of the hidden unit $\mathrm{Z}_{\mathrm{j}}$. The adjustment to the weight $\mathrm{V}_{\mathrm{ij}}$ (from input unit $\mathrm{X}_{\mathrm{i}}$ to hidden unit $\mathrm{Z}_{\mathrm{j}}$ ) is based on the factor $\delta_{\mathrm{j}}$ and the activation $\mathrm{X}_{\mathrm{i}}$ of the input unit [11].

\section{B. The new proposed system}

The new system has three main steps: data preprocessing, separating the training data set into many parts, training the neural network with each separate part.

\section{B.1 The Preprocessing of Data}

The training vectors which consist of Open-closeHigh-Low-Volume are on drastically different scales. The volume had a very large value, but the price had a small value that makes the volume a factor influencing behavior of the neural network. 
According to Guoqiang Zhang, B. Eddy Patuwo, Michael Y. Hu [12], data normalization is performed before the training process begins due to the fact that non-linear activation functions tend to squash the output of a node either in $(0,1)$ or $(-1,1)$. As a result, a way is needed to transfer the output of the neural network back to the original data range. Even in the case a linear output function, is used, it is also stated that normalizing the inputs as well as the outputs has many advantages. Some of them avoid computational problems, so all data input must be normalized from 0 to 1 by using Max-Min normalization equation [19-23].

$$
\text { Normalized data }=\frac{\text { data }- \text { min value }}{\text { max value }- \text { min value }}
$$

\section{B.2 Separate data}

The historical data of the stock, which is 7 years from 2007 to 2014, used in training the neural network, will be divided into three equal parts. Two parts are used in training two neural networks; then the third part is used in testing the previous two networks and collecting the results and considering them as input to a third network. This network represents the head of the hierarchical order of the neural networks. After that, the system will be tested through the prices of the stocks of the years 2015, 2016 and 2017. Finally, the data of the stocks, in use, will be divided into four equal parts and the previous steps will be repeated and also divided into five or six equal parts.

\section{B.3 Training network}

In this step all the results are noted to reach a better form of stocks. All the neural networks have been trained on 1000, 5000, 10000 and 20000 epoch and have been changed from 2 to 10 hidden layer and so on.

\section{RESULTS AND ANALYSIS}

Results of two shares in the Egyptian stock were recorded to different structure of neural network, different number of training epoch and different number of neural network, as the base of hierarchal shape and the result was:

In the company of Arabia and Polivara Lalghazle Wa Elnasig (APSW), minimum MSE at 3 input network 6 hidden unit 1000 epoch with MSE value $=0.0017$ and time of training in serial mode $=63.6710 \mathrm{sec}$ and time of training in Parallel mode $=30.2684 \mathrm{sec}$. The comparison between predicted price and real price of share are plotted in and all results are shown in table 1.

In Alahely Laltanmia Wa Alstasmar (AFDI), minimum MSE 3 input network 6 hidden unit 5000 epoch with MSE value $=0.0184$ and time of training in serial mode $=$ $64.0604 \mathrm{sec}$ and time of training in Parallel mode = $25.7470 \mathrm{sec}$. the comparison between predicted price and real price of share are plotted in figure 3 and all results are shown in table 1 .

When Parallel computing is used in training the neural network, the overall time of training of neural network is reduced and when the training set is divided among many networks, the number of vectors which are trained to each network is reduced and this in turn reduces error and the complexity of the network which and training time.

As appear in table 3 and table 4 the prediction quality is efficient for all test shares not only in the prediction values, but also in training time of the network.

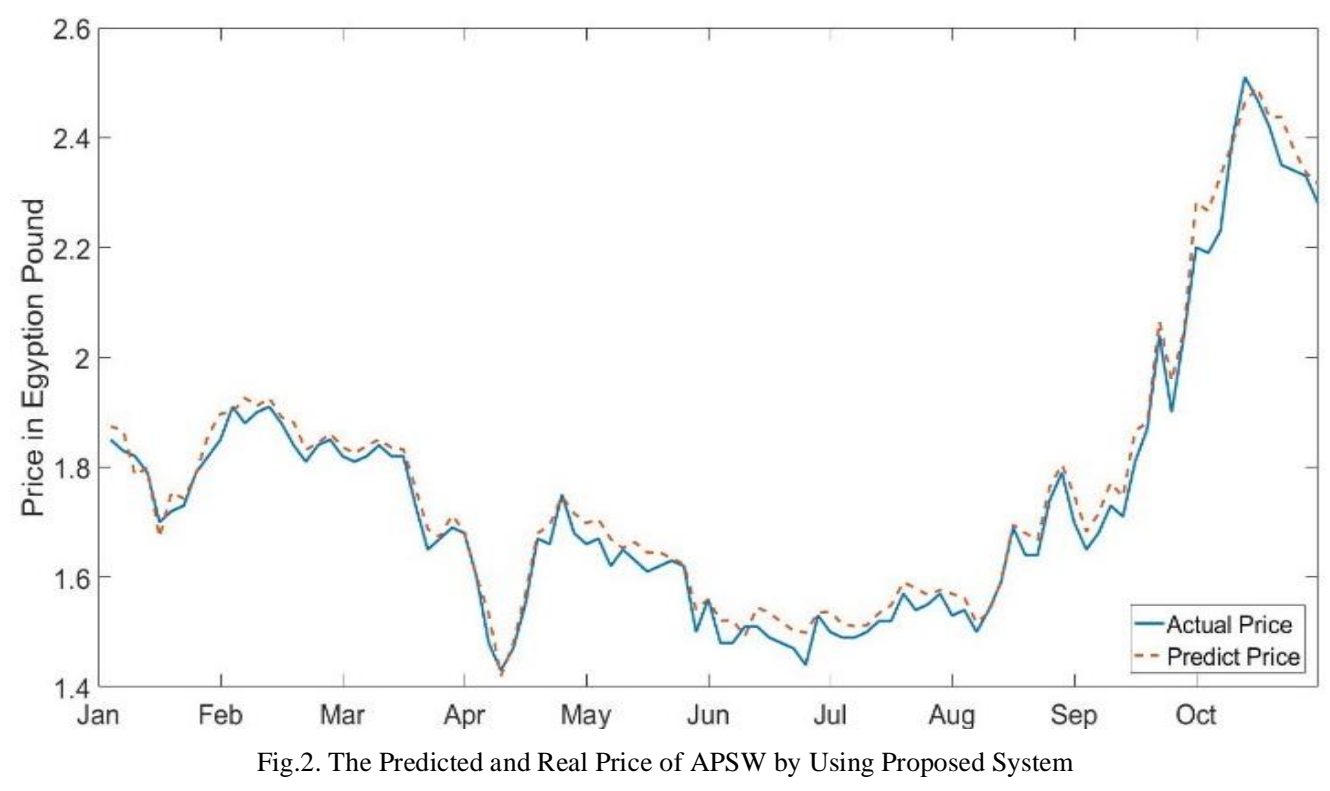


Table 1. MSE of Predicted Value of APSW by Using Proposed System

\begin{tabular}{|c|c|c|c|c|c|}
\hline & Number of Epoch & 2 Input Network & 3 Input Network & 4 Input Network & 5 Input Network \\
\hline \multirow{4}{*}{$\begin{array}{l}2 \text { Hidden } \\
\text { Units }\end{array}$} & 1000 epoch & 0.001882 & 0.002375 & 0.136248 & 0.007366 \\
\hline & 5000 epoch & 0.001861 & 0.002623 & 0.002899 & 0.002321 \\
\hline & 10000 epoch & 0.00188 & 0.0024 & 0.013596 & 0.005476 \\
\hline & 20000 epoch & 0.001935 & 0.002651 & 0.002661 & 0.002108 \\
\hline & & & & & \\
\hline \multirow{4}{*}{$\begin{array}{l}3 \text { Hidden } \\
\text { Units }\end{array}$} & 1000 epoch & 0.001879 & 0.002209 & 0.002537 & 0.007513 \\
\hline & 5000 epoch & 0.002153 & 0.001779 & 0.013517 & 0.01433 \\
\hline & 10000 epoch & 0.001911 & 0.002061 & 0.006972 & 0.039386 \\
\hline & 20000 epoch & 0.001854 & 0.002272 & 0.00246 & 0.019161 \\
\hline \multirow{5}{*}{$\begin{array}{l}4 \text { Hidden } \\
\text { Units }\end{array}$} & & & & & \\
\hline & 1000 epoch & 0.002366 & 0.002752 & 0.005151 & 0.004487 \\
\hline & 5000 epoch & 0.001987 & 0.003541 & 0.002075 & 0.011632 \\
\hline & 10000 epoch & 0.002128 & 0.001735 & 0.002548 & 0.008316 \\
\hline & 20000 epoch & 0.00215 & 0.005418 & 0.182045 & 0.002523 \\
\hline \multirow{4}{*}{$\begin{array}{l}5 \text { Hidden } \\
\text { Units }\end{array}$} & 1000 enoch & 0002345 & 0002227 & 0359413 & 0003696 \\
\hline & 5000 epoch & 0.003066 & 0.005668 & 0.00276 & 0.016487 \\
\hline & 10000 epoch & 0.002495 & 0.002698 & 0.092086 & 0.020651 \\
\hline & 20000 epoch & 0.002528 & 0.00317 & 0.002366 & 0.001883 \\
\hline & & & & & \\
\hline \multirow{4}{*}{$\begin{array}{l}6 \text { Hidden } \\
\text { Units }\end{array}$} & 1000 epoch & 0.00209 & 0.001724 & 0.00266 & 0.002785 \\
\hline & 5000 epoch & 0.002092 & 0.00327 & 0.010587 & 0.004059 \\
\hline & 10000 epoch & 0.003952 & 0.001908 & 0.003677 & 0.0092 \\
\hline & 20000 epoch & 0.004079 & 0.008066 & 0.001879 & 0.003479 \\
\hline & & & & & \\
\hline \multirow{4}{*}{$\begin{array}{l}7 \text { Hidden } \\
\text { Units }\end{array}$} & 1000 epoch & 0.005634 & 0.002191 & 0.001838 & 0.036597 \\
\hline & 5000 epoch & 0.002162 & 0.002363 & 0.008796 & 0.12004 \\
\hline & 10000 epoch & 0.002852 & 0.050816 & 0.002419 & 0.004452 \\
\hline & 20000 epoch & 0.004569 & 0.002829 & 0.003665 & 0.199039 \\
\hline \multirow{4}{*}{$\begin{array}{l}8 \text { Hidden } \\
\text { Units }\end{array}$} & 1000 epoch & 0.005034 & 0.00219 & 0.01628 & 0.008163 \\
\hline & 5000 epoch & 0.003846 & 0.020369 & 10.66238 & 0.003617 \\
\hline & 10000 epoch & 0.003093 & 0.003336 & 0.047447 & 9.359952 \\
\hline & 20000 epoch & 0.001986 & 0.001737 & 0.033 & 0.065703 \\
\hline & & & & & \\
\hline \multirow{4}{*}{$\begin{array}{l}9 \text { Hidden } \\
\text { Units }\end{array}$} & 1000 epoch & 0.009158 & 0.011002 & 99.29887 & 0.050166 \\
\hline & 5000 epoch & 0.003107 & 0.031303 & 0.009273 & 0.00524 \\
\hline & 10000 epoch & 0.260278 & 0.003504 & 1.610167 & 0.364813 \\
\hline & 20000 epoch & 0.003906 & 0.002762 & 0.953 & 0.883598 \\
\hline \multirow{4}{*}{$\begin{array}{l}10 \text { Hidden } \\
\text { Units }\end{array}$} & 1000 epoch & 0.002958 & 0.016061 & 0.024339 & 0.190392 \\
\hline & 5000 epoch & 0.002146 & 0.020701 & 0.311 & 0.2001 \\
\hline & 10000 epoch & 0.003384 & 0.102616 & 0.0464 & 0.003196 \\
\hline & 20000 epoch & 0.003378 & 0.002606 & 0.788075 & 0.068603 \\
\hline
\end{tabular}


Table 2. MSE of Predicted Value of AFDI by Using Proposed System

\begin{tabular}{|c|c|c|c|c|c|}
\hline & Number of Epoch & 2 Input Network & 3 Input Network & 4 Input Network & 5 Input Network \\
\hline \multirow{4}{*}{$\begin{array}{l}2 \text { Hidden } \\
\text { Units }\end{array}$} & 1000 epoch & 0.021947 & 0.019404 & 0.028204 & 0.027051 \\
\hline & 5000 epoch & 0.020336 & 0.021537 & 0.041995 & 0.024271 \\
\hline & 10000 epoch & 0.024133 & 0.020696 & 0.028393 & 0.020078 \\
\hline & 20000 epoch & 0.021651 & 0.177448 & 0.02281 & 0.019571 \\
\hline \multirow{4}{*}{$\begin{array}{l}3 \text { Hidden } \\
\text { Units }\end{array}$} & 1000 enoch & 0021714 & 0109878 & 0.0237 & 0.036911 \\
\hline & 5000 epoch & 0.022819 & 0.84517 & 0.10413 & 0.020288 \\
\hline & 10000 epoch & 0.024156 & 0.022253 & 0.025612 & 0.022312 \\
\hline & 20000 epoch & 0.024428 & 0.020607 & 0.018711 & 0.033687 \\
\hline & & & & & \\
\hline \multirow{4}{*}{$\begin{array}{l}4 \text { Hidden } \\
\text { Units }\end{array}$} & 1000 epoch & 0.031942 & 0.020242 & 0.018601 & 0.033454 \\
\hline & 5000 epoch & 0.021581 & 0.019363 & 0.022332 & 0.026332 \\
\hline & 10000 epoch & 0.022756 & 0.024268 & 0.023732 & 0.180472 \\
\hline & 20000 epoch & 0.028871 & 0.023095 & 0.031151 & 0.017572 \\
\hline & & & & & \\
\hline \multirow{4}{*}{$\begin{array}{l}5 \text { Hidden } \\
\text { Units }\end{array}$} & 1000 epoch & 0.028584 & 0.044131 & 0.026302 & 0.032294 \\
\hline & 5000 epoch & 0.030403 & 0.022422 & 0.019004 & 0.250389 \\
\hline & 10000 epoch & 0.022891 & 0.025216 & 0.02931 & 0.031126 \\
\hline & 20000 epoch & 0.030691 & 0.029175 & 0.170235 & 0.069502 \\
\hline \multirow{5}{*}{$\begin{array}{l}6 \text { Hidden } \\
\text { Units }\end{array}$} & 1000 omoch & 0026276 & 0010538 & 006047 & 002048 \\
\hline & $5000-1$ & 0021687 & 0010200 & 0031878 & 004001 \\
\hline & S000 еросп & 0.021081 & 0.018398 & $0.0318 / 8$ & 0.044291 \\
\hline & 10000 epoch & 0.021819 & 0.026271 & 0.022179 & 0.15899 \\
\hline & 20000 epoch & 0.024998 & 0.043651 & 0.055673 & 0.133115 \\
\hline & & & & & \\
\hline \multirow{4}{*}{$\begin{array}{l}7 \text { Hidden } \\
\text { Units }\end{array}$} & 1000 epoch & 0.02627 & 0.119427 & 0.028744 & 0.092296 \\
\hline & 5000 epoch & 0.025249 & 3012.623 & 0.083991 & 0.035706 \\
\hline & 10000 epoch & 0.025551 & 0.022322 & 0.025876 & 1.536513 \\
\hline & 20000 epoch & 0.021602 & 0.021704 & 0.033393 & 10428.53 \\
\hline \multirow{4}{*}{$\begin{array}{l}8 \text { Hidden } \\
\text { Units }\end{array}$} & 1000 epoch & 0.024245 & 0.024205 & 0.060769 & 0.08655 \\
\hline & 5000 epoch & 0.027045 & 0.029882 & 0.040683 & 0.09766 \\
\hline & 10000 epoch & 0.046768 & 0.95972 & 0.027265 & 0.6925 \\
\hline & 20000 epoch & 0.056252 & 0.028679 & 0.031184 & 0.030349 \\
\hline & & & & & \\
\hline \multirow{4}{*}{$\begin{array}{l}9 \text { Hidden } \\
\text { Units }\end{array}$} & 1000 epoch & 0.439601 & 0.075547 & 0.045314 & 0.484801 \\
\hline & 5000 epoch & 0.074946 & 0.020189 & 0.063909 & 0.118255 \\
\hline & 10000 epoch & 0.0604 & 0.022808 & 0.027645 & 0.107475 \\
\hline & 20000 epoch & 0.030325 & 0.190639 & 0.02156 & 0.102232 \\
\hline \multirow{4}{*}{$\begin{array}{l}10 \text { Hidden } \\
\text { Units }\end{array}$} & 1000 epoch & 0.025853 & 0.095796 & 0.032715 & 0.687401 \\
\hline & 5000 epoch & 0.029063 & 0.597869 & 0.082724 & 0.04836 \\
\hline & 10000 epoch & 0.02373 & 0.027377 & 0.292866 & 0.487553 \\
\hline & 20000 epoch & 0.043628 & 0.656717 & 0.039819 & 0.377600 \\
\hline
\end{tabular}




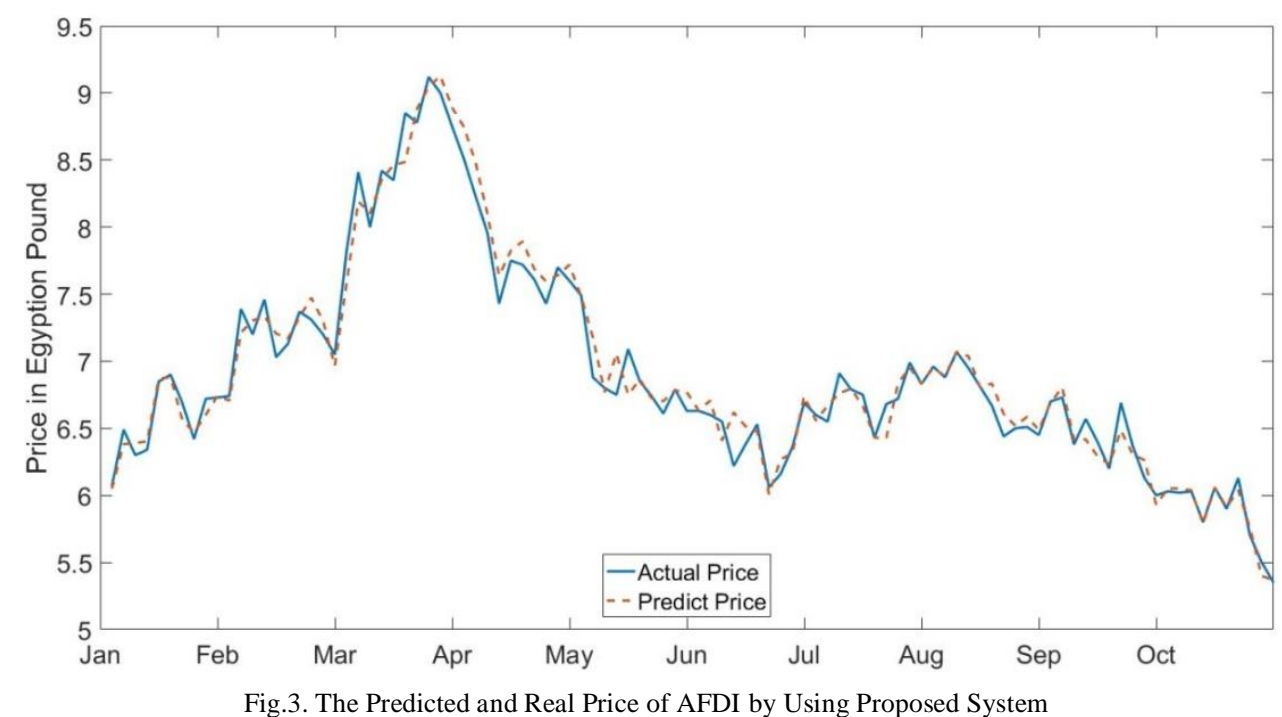

Table 3. Comparison in MSE between Traditional neural network and Heretical structure of neural network

\begin{tabular}{|c|c|c|}
\hline & Traditional neural network & Heretical structure of neural network \\
\hline Arabia Lahalig Alactan (ACGC) & 0.014335 & 0.004493 \\
\hline Arabia Wa Polivara Lalghazle Wa Elnasig (APSW) & 0.0083 & 0.0017 \\
\hline Alahely Laltanmia Wa Alstasmar (AFDI) & 0.101413 & 0.0184 \\
\hline
\end{tabular}

Table 4. Comparison in training time between Traditional neural network and Heretical structure of neural network in serial and parallel mode

\begin{tabular}{|c|c|c|c|}
\hline & Traditional neural network & $\begin{array}{c}\text { Heretical structure of neural } \\
\text { network in serial mode }\end{array}$ & $\begin{array}{c}\text { Heretical structure of neural } \\
\text { network in parallel mode }\end{array}$ \\
\hline Arabia Lahalig Alactan (ACGC) & $34.8950 \mathrm{sec}$ & $41.6383 \mathrm{sec}$ & $32.8905 \mathrm{sec}$ \\
\hline $\begin{array}{c}\text { Arabia Wa Polivara Lalghazle } \\
\text { Wa Elnasig (APSW) }\end{array}$ & $181.6082 \mathrm{sec}$ & $63.6710 \mathrm{sec}$ & $30.2684 \mathrm{sec}$ \\
\hline $\begin{array}{c}\text { Alahely Laltanmia Wa } \\
\text { Alstasmar (AFDI) }\end{array}$ & $178.5513 \mathrm{sec}$ & $64.0604 \mathrm{sec}$ & $25.7470 \mathrm{sec}$ \\
\hline
\end{tabular}

\section{CONCLUSION}

The prediction quality is efficient for all test shares not only in the prediction values, but also in training time of the network. The neural network is trained within seven year on data points, and tested within three year for all the share and the enhancement in MSE was $68.5 \%$ in share of Arabia Lahalig Alactan (ACGC), $79.5 \%$ in share of Arabia Wa Polivara Lalghazle Wa Elnasig (APSW) and $81 \%$ in share of Alahely Laltanmia Wa Alstasmar (AFDI). Also, the enhancement in training time as $5.7 \%$ in share of Arabia Lahalig Alactan (ACGC), 83.3\% in share of Arabia Wa Polivara Lalghazle Wa Elnasig (APSW) and $85.6 \%$ in share of Alahely Laltanmia Wa Alstasmar (AFDI). Finally, heretical structure of neural network is proven to be very powerful when used for prediction problems of shares daily prices. It improves the Mean Square Error between the real value of price and the predicted value from the system, also it reduces the training time of network when it trains in parallel and also it reduces the complicated structure of neural network because each network learns less data input.

Finally in future we need to test the existing methods with more data as the keep coming. We want to ensure that the results we got it is not just a random event. We have to test with even more shares to make sure that our model can generalize.

Another thing we can do is check how our model works with stocks outside the EGX 30 index. We can try train and evaluate out model with stocks that belong to smaller companies that do not have as many transactions as the big ones. In that case we will be able to see if we can expand our work to other stocks and may be even other stock markets as well

\section{REFERENCES}

[1] G. Appel, "Technical Analysis Power Tools for Active Investors". Financial Times, Prentice Hall, 1999,pp. 166167.

[2] M. Haugh, "Overview of Monte Carlo Simulation, Probability Review and Introduction to Matlab". IEOR E4703, 2004, pp. 1 - 11.

[3] F. Emmanuel, "Performance Measure of Binomial Model for Pricing American and European options", 2014. OnlineDOI: 10.11648/j.acm.s.2014030601.14

[4] G. Dreyfus, "Neural Networks Methodology and Applications", Springer ,pp. 203-230

[5] D. Utomo "Stock Price Prediction Using Back Propagation Neural Network based on Gradient Descent with Momentum and Adaptive Learning Rate", Journal of Internet Banking and Commerce, 2017.

[6] R. Trifonov "Artificial Neural Network Intelligent Method 
for Prediction" OnlineDOI: 10.1063/1.4996678.

[7] T. Lin "Hybrid Neural Networks for Learning the Trend in Time Series", In Proceedings of the Twenty-Sixth International Joint Conference on Artificial Intelligencem, 2017.

[8] Hiransha, "Stock Market Prediction Using Deep-Learning Models", International Conference on Computational Intelligence and Data Science, 2018, pp 1351-1362.

[9] M. Mitchell Machine learning, The McGraw-Hill, 1997.

[10] D. Rumelhart "Learning Representations by Backpropagating Errors". Cognitive modeling, 1988.

[11] L Fausett "Fundamentals of Neural Networks: Architectures, Algorithms And Applications" Vol 1, Pearson, 1993.

[12] Forecasting With Artificial Neural Networks: The State of the Art Online DOI: 10.1016/S0169-2070(97)00044-7.

[13] S. Haykin, "Neural Networks and Learning Machines", Pearson, 2011.

[14] J. Dayhoff, "Neural Network Architectures", VanNostrand Reinholt, 1990.

[15] J. Person, "Complete Guide to Technical Trading Tactics: How to Profit Using Pivot Points, Candlesticks \& Other Indicators", Wiley, 2004, pp 144-145.

[16] J.Murphy,"Technical Analysis of the Financial Markets: A Comprehensive Guide to Trading Methods and Applications", New York Institute of Finance, 1999, pp 247-248.

[17] M.Pring, "Technical Analysis Explained The Successful Investor's Guide to Spotting Investment Trends and Turning Points", McGraw-Hill Education, 2014,pp 340370.

[18] Chen S., Stock Prediction Using Convolutional Neural Network OnlineDOI:10.1088/1757-899X/435/1/012026.

[19] L. Shalabi, Z. Shaaban, and B. Kasasbeh., "Data Mining: A Preprocessing Engine" Computer Science, vol. 2.2006, pp. 735-739.

[20] P. Panda, N. Subhrajit and P. Jana, "A Smoothing Based Task Scheduling Algorithm for Heterogeneous MultiCloud Environment" $3^{\text {rd }}$ IEEE International Conference on Parallel, Distributed and Grid Computing (PDGC), IEEE, Waknaghat, 2014.

[21] P. Panda and P. Jana, "Efficient Task Scheduling Algorithms for Heterogeneous Multi-cloud Environment" The Journal of Supercomputing, Springer, 2015.

[22] P. Panda and P. Jana, "An Efficient Task Scheduling Algorithm for Heterogeneous Multi-cloud Environment",

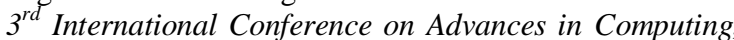
Communications \& Informatics (ICACCI), IEEE, Noida, 2014, pp. $1204-1209$.

[23] P. Panda and P. Jana,. "A Multi-Objective Task Scheduling Algorithm for Heterogeneous Multi-Cloud Environment", International Conference on Electronic Design, Computer Networks and Automated Verification (EDCAV), IEEE, Meghalaya, 2015.

[24] K. Gurney, "An Introduction to Neural Networks", CRC Press, 2003.

[25] R Edwards, J. Magee and W. Bassetti, "Technical Analysis of Stock Trends" Online DOI:10.4324/9781315115719

\section{Authors' Profiles}

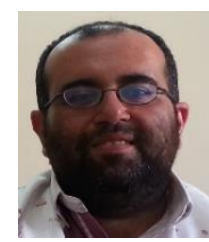

Ahmed S. Mahedy received his BSc in 2000 from Port Said University.

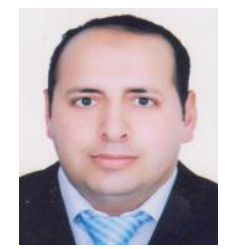

Abdelazeem A. Abdelsalam is an Assistant Professor at Suez Canal University, Egypt. He was a post doctorate fellow at University of Ontario Institute of Technology (UOIT), Canada. He received his B.Sc., M.Sc. and Ph. D. degrees in Electrical Engineering from Suez Canal University, Egypt, in 2001, 2005 and 2011, respectively. His current research areas include power quality issues, FACTS technology, distributed energy resources interface and control and application of artificial intelligent techniques on power systems.

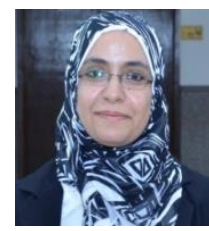

Reham H. Mohammed received her BSc in 2005 from Mansoura University, MSc in 2010 Mansoura University, and PhD in 2016 from Suez Canal University. She worked as lecturer at Department of Electrical Engineering, University of Suez Canal, Egypt She has more than 10 scientific research papers published in prestigious international journals.

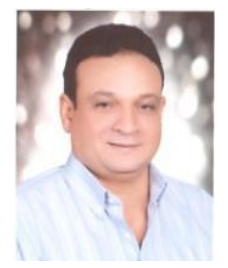

Ebrahim Elnahri, Assistant Professor, Faculty of Engineering, Department of Computer and Control System, Manager of Quality Assurance Unit, Faculty of Engineering, University of Port Said. Egypt, $\mathrm{He}$ was working at University of Tabuk, Faculty of computers and Information Technology for 6 years.

How to cite this paper: Ahmed S. Mahedy, Abdelazeem A. Abdelsalam, Reham H. Mohamed, Ibrahim F. El-Nahry, "Utilizing Neural Networks for Stocks Prices Prediction in Stocks Markets", International Journal of Information Technology and Computer Science(IJITCS), Vol.12, No.3, pp.1-7, 2020. DOI: 10.5815/ijitcs.2020.03.01 